\title{
Saga of Covid 19 Vaccination
}

\section{Suresh $\mathrm{K}^{*}$}

Public Health Consultant, India

*Corresponding author: Suresh K, MD, DIH, FIAP, FIPHA, FISCD, Public Health Consultant, India, Email: ksuresh.20@gmail.com

\section{Review Article}

Volume 4 Issue 3

Received Date: April 12, 2021

Published Date: May 11, 2021

DOI: $10.23880 /$ jqhe-16000223

\section{Abstract}

Covid 19 Pandemic hit 219 countries and territories of the world since the first human cases of COVID-19 were identified in Wuhan, the capital of the province of Hubei in China in December 2019. Initial Pandemic management strategy included Testing and confirming by laboratory tests namely RTPCR / Rapid antigen tests, treating with antivirals and corticosteroids and complications as they manifested, tracking contacts, promoting use of masks, handwashing, and respiratory etiquette. The world scientist delivered several excellent options within a year a significant achievement in the Public Health History. Now every country is tripping over itself in trying to get vaccinated their population as an additional strategy to achieve immunity and prevent grievous effects both on health and economy of the country. But there are also attempts to cut the line, and cutthroat competition with an adverse impact on low and middle socio-economic countries.

Vaccinating a huge adult population is a complex process for want of experience even in advanced countries, especially for newly Introduced vaccines like COVID-19 vaccination and reaching the required coverage of at least $70 \%$ of the population for developing herd immunity will take 1-2 years. It is desirable for countries to share lessons learned from rollout to benefit from each other's experiences. Initial experimentations with varieties of vaccine available, vaccine logistics, different strategies to scale up the expansion starting from high-risk population, aftereffects following Immunization (AEFI) and their management as the vaccines are released for emergency use. This should be taken as an opportunity for strengthening broader immunization and health systems to resolve existing weaknesses and to meet future needs for robust essential health services. Appropriate communication on the benefit-risk profile of COVID-19 vaccines remains crucial to maintain confidence in immunization programmes and to avoid vaccine hesitancy.

Vaccine hesitancy takes different forms for different people. For some, there is blanket scepticism and for others, it is a more specific distrust of Big Pharma. There will be some others who are naturally cautious of anything new and sudden. They need a lot of re-assurance before being able to accept the vaccine. Social media is giving wrong information about the vaccine and there are many avid followers of the same.

Immunization is a global public good, with a significant segment of non- enthusiastic people. Therefore, every country must ensure universal and equitable access to safe, efficacious, and affordable vaccines, optimal use of vaccines, medicines, and diagnostics.

While we accelerate the efforts of vaccinations, it is vital that even after vaccination with both doses everyone must wear masks, practice social distancing, and wash their hands when around those who have not been vaccinated

This article reviews issues related Covid 19 Vaccines, Vaccination, AEFI, acceptance, Hesitancy, and reason for the same worldwide with special reference in the Indian context. 


\section{Journal of Quality in Health care \& Economics}

Materials \& Methods: This manuscript has used Global vaccination data, reports on adverse reactions as they occurred in different media, Government of India, Ministry of Health and Family Welfare and State government reports, media coverage on challenges of online registration, handling of vaccination in government and private facilities, AEFI reports and rumour on social media.

Background: Covid 19 Pandemic hit the entire world since the first human cases of COVID-19 were identified in Wuhan, the capital of the province of Hubei in China in December 2019. On January 30,2020, the World Health Organization declared the coronavirus outbreak as a Global Public Health Emergency. On January 31, the first 2 novel coronavirus cases in the UK, the first 2 cases in Russia, and the first case in Sweden and in Spain were reported. Canada reported its 4th case. On the same day, the United States declared Coronavirus a Public Health Emergency. The Pandemic of SARS COV 2 popularly known as 'Corona Virus19' has affected 219 countries and territories around the world as of today. The COVID-19 pandemic is the biggest challenge to the global community since the 1940s.

Around 134,641,215 cases and 2,917,995 deaths have been reported as on the World Health Day (7th April) of 2021. Czechia tops the list of countries worst affected with 146,691 cases per million population, followed by USA 95,392, Israel 90,854, whereas India has just 9393 cases per million population. Czechia has record Covid 19 death rates of 2,575/million population followed by Belgium 2,008, Italy 1,869, UK-1,863, USA 1,726, and India 121 per million population. In terms of the population tested for Covid 19 infections UK stands out with 1,871,439 /million, USA 229,231, France 1,001,532, Czechia 1,206,433, and India just 177,643 test per million population. China where the pandemic originated has reported 160,000,000 tests with a rate of 111,163 test/million population, a total of 90,252 cases with a rate of 63 cases and 3 deaths per million populations so far.

The soaring numbers with the rolling 7 days average of 337 cases per million population in Italy followed by 203 in Germany,198 in USA,141 in Canada, 60 in UK and 47 in India as of first week of April 2021, that we are seeing today will continue to rise unless there is a serious attempt to get everyone vaccinated. Initial Pandemic strategy included Testing, confirmation by RTPCR tests, and treatment of confirmed cases with antivirals and corticosteroids and complications as they arose in well-equipped hospitals, Isolation of cases either in homes or institutions, screening of international travellers, tracking and testing primary and secondary contacts, quarantining suspected persons. Promoting using masks, handwashing, and Respiratory etiquette were the main stay of controlling the spread of the pandemic. After a full year's efforts multiple effective vaccines have been developed. But in a hurry to benefit their own population each country of the world is tripping over itself to get vaccinated. There are attempts to cut line, frustration breeding among poorer countries at finding oneself low down on the list of who is eligible to get the vaccine on sheer economic consideration not the humanitarian.

There is a significant segment that does not seem enthusiastic. Vaccine hesitancy takes different forms for different people. For some, there is blanket scepticism about any mainstream narrative and a nagging suspicion that some conspiracy is afoot. For others, it is a more specific distrust on Big Pharma's. There are those that are naturally cautious of anything new and sudden and need a lot of re-assurance before being able to accept the vaccine. Some have the wrong information about the vaccine, being avid followers of the kind of knowledge most prevalent on WhatsApp, Twitter, Facebook etc. Still there are others who are passive and indifferent and believe that neither the Covid 19 infection nor the vaccine has nothing to do with them.

Keywords: Vaccination in Adults; Emergency Use Clearance of Vaccines; Vaccine Hesitancy; Adverse Events Following Immunization (AEFI); Vaccine Holding 


\section{Introduction}

Reaching large number of adults for any vaccination is a complex phenomenon. Introduction of COVID-19 vaccination following a fulminating pandemic may appear easy but the none of the vaccines available have proved their effectiveness in the community so far, though every manufacturer claims an efficiency of $70-95 \%$. All the 8 candidate vaccines are released for emergency use. Therefore, it is valuable for countries to share lessons learned from COVID-19 vaccine rollout to benefit from each other's experiences. This effort will benefit from strong political will; comprehensive communication and community engagement; robust microplanning; interoperable digital tools which allow registration, safety monitoring, vaccine stock management, follow-up reminders, and vaccination certificates; National and local efforts to engages partners and stakeholders, including the private sector are key for the success. Introduction of COVID-19 vaccination should be taken as an opportunity for strengthening broader immunization and health systems to resolve existing weaknesses and to meet future needs for robust essential health services. Appropriate communication on the benefit-risk profile of COVID-19 vaccines remains crucial to maintain confidence in immunization programmes and to avoid vaccine hesitancy.

As vaccine-preventable infectious diseases continue to decline, people have become increasingly concerned about the risks associated with vaccines. Furthermore, technological advances and continuously increased knowledge about vaccines have led to investigations focused on the safety of existing vaccines which have sometimes created a climate of concern. Adverse event following immunization (AEFI) is any untoward medical occurrence which follows immunization, and which does not necessarily have a causal relationship with the usage of the vaccine. If not rapidly and effectively dealt with, can undermine confidence in a vaccine and ultimately have dramatic consequences for immunization coverage and disease incidence. Vaccine-associated adverse events may affect healthy individuals. It should be promptly identified, researched and appropriate action taken.

The Vaccine hesitancy coming from a tendency to privilege personal anecdotal experience like the doubt sowed by instances of fully vaccinated people getting infected again. If vaccine efficacy is as high as $95 \%$, even then some people will get sick. Given the large base of those vaccinated, this small number in terms of percentage will translate into a large physical number. However, the emergence of such cases, particularly if they occur in people one knows of, is psychologically difficult to reconcile with the expectation of being protected. Many stories of sundry side-effects again hardly ever serious or unanticipated, get disproportionate play. The way some vaccines have communicated their progress for example, AstraZeneca has scored self-goals and jeopardised the reputation of what otherwise looks like an excellent vaccine. The latest imbroglio around its claimed efficacy of $79 \%$, the public rebuke that it received from US regulators and some other European countries is difficult to comprehend. Vaccine hoardings by rich countries make inaccessible to poor countries and anti-vaccination segment in west especially USA are other significant reasons for a long-drawn battle to cover desired proportion of population to get herd immunity and benefit the world at large. A lack of confidence in a home grown vaccine country could prevent India from meeting its target of vaccinating 300 million of its 1.35 billion people by August 2021 .

The vaccination drive must have twin objectives - to reduce serious cases needing hospitalisation and curtailing deaths. The country-wide data as of April 1 showed that $40 \%$ of all positive cases and $88 \%$ of deaths occurred in the age group of 45 years and above. Therefore, the prioritization listed by all countries and Govt. of India are justified.

\section{Vaccines Worldwide}

Covid 19 vaccines stimulate Both IgG and IgA antibodies. IgG confine to our internal organs like muscles, blood and the lungs and provide systemic protection. Science does not know still how IgA are produced but we know that the IgA are on the same respiratory surface as throat, lungs and digestive tract that transmit corona virus and logically may interfere in transmitting the viruses. We still must wait to get a definite answer for this logic. Many vaccines are being developed at record speed, due to years of research on related viruses and billions of dollars in investment.

To date, many COVID-19 vaccines are being rapidly developed worldwide, with thirteen candidates in Phase 3 trials, 52 tested in clinical trials, and 162 in preclinical evaluation. Here, I summarize the latest progress of 10 COVID-19 vaccines in Phase 3 trails. There are about 8 varieties of vaccine of which nearly 150 million have been used worldwide as 1 April 2021. They are Moderna- $71.34 \mathrm{~m}$, AstraZeneca/Oxford -, Pfizer/ BioNtech -78.72 m, Sonovac, Johnson \& Johnson (3.34m), Sputnik V, Covaxin, Sinopharm/ Beijing. We do notyet know whether the available vaccines will prevent infection and protect against onward transmission. Immunity persists for several months, but the full duration is not yet known. These important questions are being studied and may clarified over next one year. As of now we know that the vaccines prevent severity of the manifestations and reduce the case fatality rates. Currently available vaccines are not recommended for children under18 years, pregnant and lactating mothers. 


\section{Pfizer-BioNTech COVID-19 Vaccine}

The Pfizer-BioNTech COVID-19 vaccine has not been approved or licensed by the U.S. Food and DrugAdministration (FDA), but has been authorized for emergency use by FDA under an Emergency Use Authorization (EUA) to prevent Coronavirus Disease 2019 (COVID-19) for use in individuals 16 years of age and older. The emergency use of this product is only authorized for the duration of the declaration that the circumstances exist justifying the authorization of emergency use of the medical product. It is a messenger RNA (mRNA) vaccine that has both synthetic, or chemically produced, components and enzymatically produced components from naturally occurring substances such as proteins. The vaccine does not contain any live virus. Its inactive ingredients include potassium chloride, monobasic potassium, phosphate, sodium chloride, dibasic sodium phosphate dihydrate, and sucrose, as well as small amounts of other ingredients. It is currently used in several American and European countries but not in India, as it must be stored at $-70 \mathrm{C}$ and can only be moved a limited number of times.

A recent study released on 10 April 21 in Israel that compared almost 400 people who had tested positive for Covid with South African variant, 14 days or more after they received one or two doses of the vaccine, against the same number of unvaccinated patients with the disease. Among patients who had got two doses of the vaccine, the variant's prevalence rate was 8 times higher than those unvaccinated $5.4 \%$ versus $0.7 \%$., suggesting that the vaccine is less effective against the South African variant, The South African variant, B.1.351, was found to make up about $1 \%$ of all the cases across all the people studied (Table 1).

\begin{tabular}{|c|c|c|c|c|c|}
\hline Company & Type & Doses & $\begin{array}{c}\text { How } \\
\text { Effective }\end{array}$ & Storage & Cost \$/Dose \\
\hline $\begin{array}{c}\text { Oxford University/AstraZeneca/SII/ } \\
\text { Pune India }\end{array}$ & $\begin{array}{c}\text { Viral Vector (genetically } \\
\text { modified virus) }\end{array}$ & 2 injections & $60-90 \%$ & $2-8^{\circ}$ Celsius & 4 \\
\hline Moderna & $\begin{array}{c}\text { RNA (part of virus } \\
\text { genetic code) }\end{array}$ & 2 injections & $95 \%$ & $\begin{array}{c}-20^{\circ} \text { cup of } \\
6 \text { months }\end{array}$ & 33 \\
\hline Pfizer-BioNTech & RNA & 2 injections & $95 \%$ & $-70^{\circ} \mathrm{C}$ & 20 \\
\hline Gamaleya (Spuntik V) & Viral Vector & 2 injections & $92 \%$ & $2-8^{\circ}$ Celsius & 10 \\
\hline Covaxin (HYD india) (Bharat Biotech) & $\begin{array}{c}\text { Inactivated Corona Virus } \\
\text { Isolated in NII }\end{array}$ & 2 injections & $90 \%$ & $2-8^{\circ}$ Celsius & 4 \\
\hline
\end{tabular}

Table 1: Some of the Covid 19 Vaccines Comparison.

- mRNA vaccine in India: The clinical trials of India's first indigenous mRNA vaccine candidate HGCO19 to assess its safety and immunogenicity against Covid-19 is expected to begin in Pune and Kolhapur. The technology is like the one used for the Pfizer vaccine. This vaccine has been developed Gennova Biopharmaceuticals Limited in collaboration with HDT Biotech Corporation, Seattle, USA.

- Moderna Vaccine: mRNA-1273 is the working name of Moderna's vaccine. The mRNA in the name means messenger RNA, which carries the genetic formula for the coding of a specific protein for the most distinguishing feature of the SARS-CoV2 - the spike protein - which is also the appendage that the virus uses to enter the cell and replicate. The vaccine, when injected into a person, codes for the spike protein, the body learns what the virus looks like and arms itself with the antibodies that are required to act against it.

- Chinese Vaccines: The other alum-adjuvanted inactivated Covid-19 vaccines are BBIBPCorV and CoronaVac developed by Chinese players Sinopharm and Sinovac. "Overall, Algel-IMDG-adjuvanted BBV152 was safe, immunogenic, and able to induce Th1-based T-cell responses and, therefore, could be a potentially superior vaccine over the alum-adjuvanted inactivated Covid-19 vaccines. Provincial Centre for Disease Control and Prevention had claimed in a comment in The Lancet Infectious Diseases. Beijing had distributed hundreds of millions of doses abroad while trying to promote doubt about the effectiveness of the Pfizer-BioNTech vaccine made using the previously experimental messenger RNA, or mRNA, process. Chinese top disease control Center has admitted on 10 April2021 the fact that Chinese Covid vaccines, effectiveness is low as they do not have very high protection rate. The effectiveness of a Sinovac vaccine was found to be as low as $50.4 \%$ by researchers in Brazil, nearer the $50 \%$ threshold at which experts find a vaccine is useful. By comparison, the Pfizer vaccine has been found to be $97 \%$ effective AP.

- Covishield the Oxford-AstraZeneca vaccine is being manufactured locally by the Serum Institute of India, the world's largest vaccine manufacturer, producing more than 50 million doses a month. The vaccine is made from a weakened version of a common cold virus (known as 
an adenovirus) from chimpanzees. It has been modified to look more like coronavirus - although it cannot cause illness. When the vaccine is injected into a patient, it prompts the immune system to start making antibodies and primes it to attack any coronavirus infection. The jab is administered in two doses given between four and 12 weeks apart. It can be safely stored at temperatures of $2 \mathrm{C}$ to $8 \mathrm{C}$ and can be delivered in existing health care settings. This vaccine has been dogged for weeks over possible links to rare blood clots in the brain and abdomen reported from many European countries and suspending its use for young adults.

- Covaxin: Covaxin is an inactivated vaccine, making it safe to be injected into the body. Bharat Biotech used a sample of the coronavirus, isolated by India's National Institute of Virology. When administered, immune cells can still recognise the dead virus, prompting the immune system to make antibodies against the pandemic virus. The two doses are given four weeks apart. The vaccine can be stored at $2 \mathrm{C}$ to $8 \mathrm{C}$. Bharat Biotech has a stockpile of 20 million doses of Covaxin and can make 700 million doses out of its four facilities in two cities by the end of the year.

India's first indigenous Covid-19 vaccine Covaxin, codenamed BBV152, is safe, immunogenic and has no serious adverse effects, medical journal Lancet has said while publishing the vaccine's phase 2 trial results on 10 March 2021. The journal also said the vaccine, which uses an ingredient called IMDG along with alum (Algel, an aluminium salt) to boost the immune response, could be superior to other inactivated SARSCoV-2 vaccines that use only alum as adjuvant. An adjuvant is used in vaccines to evoke a stronger immune response in those being vaccinated. The drug regulator's subject expert committee (SEC) on Wednesday 13 March 21 recommended doing away with the "clinical trial mode" for its use and making it a case of regular emergency use authorisation.

- Sputnik V: Since March 21 Russia's sovereign wealth fund- Russian Direct Investment Fund (RDIF) has already tied up manufacturing of over 850 million doses of Sputnik V in India. This includes an agreement with Hyderabad-players like Hetero Biopharma for 100 million doses, Gland Pharma for 252 million doses, Virchow Biotech for 200 million doses. The Russian sovereign fund has also tied-up manufacture of 100 million doses each with Panacea Biotech and Stelis Biopharma of Bengaluru.

- Dr Reddy's for Sputnik V: Pharma industry sources say commercial manufacture of Sputnik V in India will begin only after the Drugs Controller General of India (DCGI) EUA approval to Dr Reddy's.

- Johnson \& Johnson Janssen Vaccine: Janssen vaccine is a Viral Vector vaccine manufactured by Janssen
Pharmaceuticals Companies of Johnson \& Johnson. It is the only single dose Shot currently available given in the muscle of the upper arm. This vaccine is also recommended for people aged 18 years and older. The ingredients include recombinant, replicationincompetent adenovirus type 26 expressing the SARS-CoV-2 spike protein, citric acid monohydrate, trisodium citrate dihydrate, ethanol, 2-hydroxypropyl- $\beta$ cyclodextrin (HBCD), polysorbate-80, sodium chloride. The contraindications include any severe allergic reaction to any ingredient of this vaccine. Severe allergic reactions have been reported in USA \& Europe recently. Of the four serious cases of clotting, three occurred in the US during the rollout of J\&J's vaccine from its Janssen unit. The fourth case was reported by the European Medicines Agency of one person dying in a trial. It was the first news of EMA's probe of the J\&J vaccine. US FDA has confirmed on 9 April 21 that a few individuals have suffered clots and low levels of plate in the blood after receiving J\&J vaccine. The further elaborate that both these conditions have multiple causes and at present there are no causal relationship with J\&J Vaccine. J\&J said that it was aware of the reports of blood clots and is working with regulators to assess the data and provide relevant information. European Medical Agency (EUA) has also said that at present, no clear causal relationship has been established. J\&J's single-dose jab has been approved for EU use, but a rollout is yet to begin.

The global manufacturers can manufacture enough (up to 12 billion doses) and make available by the end of the year 2021 , that could suffice for $70 \%$ of the global population. Till now, over 400 million doses have been administered in over 130 countries, but manufacturing and supply have become critical constraints for global access and equity, [Duke Global Health Innovation Center]. The challenge is if those doses were purchased and distributed equitably across the world's population. Assuming the market is primarily two-dose vaccines as of now (Johnson \& Johnson/Janssen and Chinese CanSino are the only single-dose vaccines currently available in a few countries), about 11 billion doses are required to vaccinate $70 \%$ of the world's population. This level is significant as it is frequently perceived as the threshold to approach herd immunity. But the current global data continues to show an astonishing gap between wealthy countries and low and middle-income countries in terms of who controls the lion's share of vaccines. "Covid-19 vaccine access remains a lopsided issue as wealthy countries have now purchased about 4.6 billion doses (USA alone- 1.2 billion) for its 330 million citizens. This as compared to 1 billion for the Covax pact supplying several billion people in low- and middle-income countries [1]. Some countries at all income levels cannot adequately assess and act to minimize risks of supply disruption, due to a rapidly shifting landscape 
of manufacturing capacity and supply projections abruptly expanding or contracting daily. It is believed that the demand for Covid-19 vaccines will outstrip supply over the coming months. There is little transparency in the landscape, therefore it is difficult for country leaders to assess the feasibility of delivery schedules or to understand the risks and contingencies [2].

Asserting that the effects of the COVID-19 pandemic will be felt for years, the International Monetary Fund and the World Bank have been urged by global leaders to ensure timely delivery of safe and effective vaccines across all countries. Timely delivery of safe and effective vaccines across all countries is critical to ending the pandemic, especially as new variants emerge. Developing countries need to strengthen their readiness for vaccination campaigns and develop coordinated strategies to reach vulnerable populations

\section{Vaccine's Status in India}

India currently has two approved vaccines in use, one developed by Oxford University and AstraZeneca at SII Pune and the other, a shot from local player Bharat Biotech with a total production capacity of 65-70 million doses per month. Indian Government has ordered 500 million Covishield and 10 million doses of Covaxin as of now. With several states complaining of vaccine shortage and temporarily shutting centres - at a time when the need is to increase the daily rate of jabs-the focus has intensified on the production and supply end of the chain.

Indications are that India's vaccine production is expected to be limited for a few more weeks though a stepup is expected as Serum Institute of India and Bharat Biotech ramp up capacity, some of which is expected to be ready by next month, prompting the central government to prioritise and finance production by committing purchase orders (Figures $1 \& 2$ ).

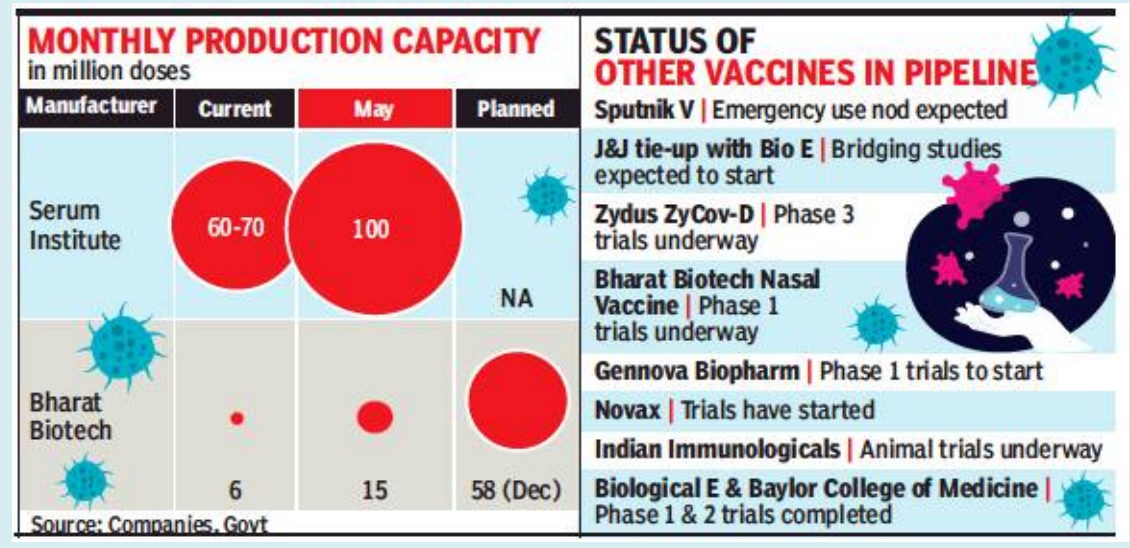

Source: Times of India, Bangalore edition 10 April 2021

Figure 1: Current Covid 19 vaccine Monthly Production Capacity and Vaccines in Pipeline in India.

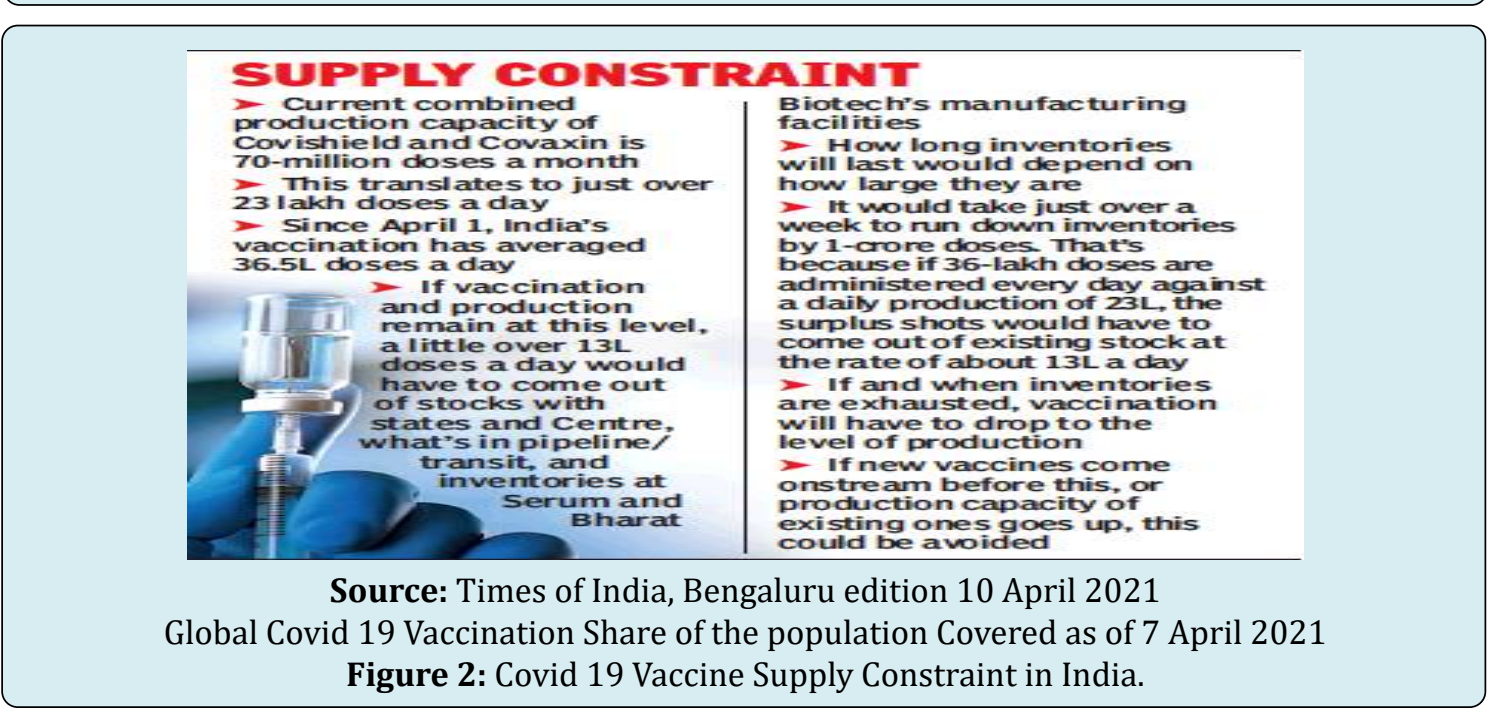


170 countries and territories have administered more than 733 million doses of a Covid-19 vaccine, by $7^{\text {th }}$ April 2021. The vaccine rollout strategy varies from country to country. Some have favoured vaccinating as many people as possible as quickly as possible, while others like India have tried to prioritize vaccinating specific vulnerable groups starting from health workers and other Covid 19 frontline warriors, followed by persons aged over 60 years and then person over 45-50 years with comorbidities and then expanding to population over 45 years and last in the line will be others.

Covid 19 vaccination started in USA on 14 December 2020 and the country has use about 171 million doses and fully protected with 2 doses about 64.5 million population so far, as per the Centres for Disease Control and Prevention Atlanta. They mainly use Pfizer vaccine and Moderna vaccine and very recently Johnson \& Johnson's single dose vaccine.
In the first week of April the country administered about 3 million doses per day based on a 7days average. So far about $20 \%$ of total population, $25 \%$ of adult $(18+)$ population and $57.4 \%$ of senior citizens have been fully protected. USA stands ahead of all countries in vaccination doses given as of 7 April 2021 with 171 million doses at least one dose. Mainland China with 149.07 million and India with 90.2 million doses follow the suit. In terms of fully vaccinated population with 2 doses Israel stand first with $55 \%$, followed by USA with $19 \%$, UK $8.5 \%$ and India less than $1 \%$ coverage had full vaccination of 2 doses. In at least one dose coverage Israel leads with $60.7 \%$, UK $45.9 \%$, USA $29.8 \%$, and India only $4.3 \%$. In terms of the population covered with 2 doses Israel tops with $56.5 \%$ coverage, followed by Chile $22.2 \%$, USA $19.3 \%$, UK $8.5 \%$, and India at poor $0.8 \%$. While Uruguay leads the proportion of people vaccinated per day with $1.07 \%$, followed by Chile $1 \%$, USA, and UK 0.87 and $0.82 \%$ India covers only $0.14 \%$ of its population (Table 2 ).

\begin{tabular}{|c|c|c|}
\hline Gibraltar & $84.9 \%$ fully vaccinated & $10.9 \%$ partially vaccinated \\
\hline Israel & $56.5 \%$ fully vaccinated & $4.6 \%$ partially vaccinated \\
\hline Chile & $22.2 \%$ fully vaccinated & $15.1 \%$ partially vaccinated \\
\hline United States & $19.3 \%$ fully vaccinated & $13.6 \%$ partially vaccinated \\
\hline Singapore & $9.2 \%$ fully vaccinated & $10.2 \%$ partially vaccinated \\
\hline Turkey & $8.7 \%$ fully vaccinated & $3.6 \%$ partially vaccinated \\
\hline United Kingdom & $8.4 \%$ fully vaccinated & $38.3 \%$ partially vaccinated \\
\hline Denmark & $7.2 \%$ fully vaccinated & $6.7 \%$ partially vaccinated \\
\hline Switzerland & $7.0 \%$ fully vaccinated & $4.5 \%$ partially vaccinated \\
\hline Spain & $6.3 \%$ fully vaccinated & $7.4 \%$ partially vaccinated \\
\hline Italy & $6.0 \%$ fully vaccinated & $7.4 \%$ partially vaccinated \\
\hline Czech Republic & $5.9 \%$ fully vaccinated & $6.0 \%$ partially vaccinated \\
\hline Germany & $5.5 \%$ fully vaccinated & $7.4 \%$ partially vaccinated \\
\hline France & $4.7 \%$ fully vaccinated & $9.3 \%$ partially vaccinated \\
\hline Russia & $3.5 \%$ fully vaccinated & $1.9 \%$ partially vaccinated \\
\hline Brazil & $2.5 \%$ fully vaccinated & $6.4 \%$ partially vaccinated \\
\hline Canada & $2.0 \%$ fully vaccinated & $14.6 \%$ partially vaccinated \\
\hline Indonesia & $1.6 \%$ fully vaccinated & $1.7 \%$ partially vaccinated \\
\hline Mexico & $1.1 \%$ fully vaccinated & $5.5 \%$ partially vaccinated \\
\hline India & $<1 \%$ fully vaccinated & $4.9 \%$ partially vaccinated \\
\hline
\end{tabular}

Source: https://www.nytimes.com/interactive/2021/world/covid-vaccinations-tracker.html

Table 2: Share of the population fully covered against Covid 19 as on 7 April 2021.

\section{Covid 19 Vaccination Status in India as of 7 April 2021}

India faces a seemingly mammoth COVID-19 vaccination challenge, but its progress so far suggests that it is well equipped to handle the task.

Government of India announced a plan to vaccinate around 300 million people by early August. To the best of my judgement based on 52 years of Public Health 
experience, seeing a part of the challenges met by the country's preparations and actions so far, I am convinced that the country is well equipped to achieve the task. India can meet the challenges of the huge scale mainly because of the commitment from our Prime Minister, Union Health Minister who he himself is a doctor and had taken in lead in polio eradication efforts through Pulse Polio Immunization in Mid 1990's. The massive manufacturing capacity, a strong public-health infrastructure with experience in vaccine delivery, an army of frontline workers, and meticulous planning are well understood by the people at the helm of the affair. These potentials are complimented by CoWIN, a digitalised platform for the effective national rollout and scaling up of the COVID Vaccine Distribution System. India has the capacity to manufacture all its COVID-19 vaccines domestically (Figure 3).

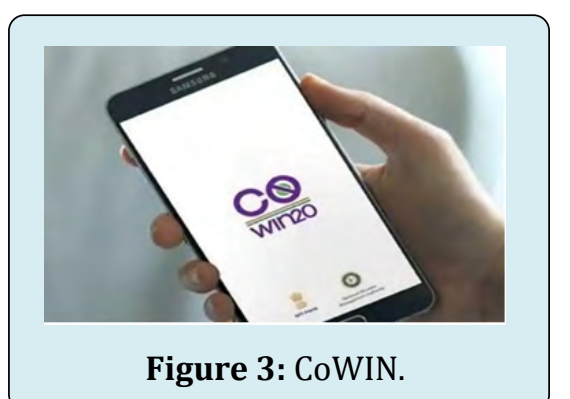

The countrywide vaccination drive was rolled out on January 16 with healthcare workers getting inoculated and vaccination of frontline workers started from February 2. This drive to vaccinate around 30 million frontline and other health workers vaccinated ten million in the first 34 days. The drive prioritised 1) healthcare and frontline workers, 2) those over the age of 60 , and 3) then those over the age of 45 and suffering from certain comorbidities and 4) all persons over 45 years since 1 April 2021.

The country is a vaccine powerhouse as it makes $60 \%$ of the world's vaccines and is home to half a dozen major manufacturers. Currently, two vaccines, named Covaxin -a whole virion inactivated SARS-CoV-2 vaccine (BBV152) and Covishield the recombinant chimpanzee Adenovirus vector vaccine (AZD1222) [ChAdOx1 $\mathrm{nCoV}-19$ ], have been granted approval from the DCGI for emergency use and are currently being administered in the Indian population. Bharat Biotech's Covaxin got clearance while the third phase of the trial was still under way, sparking scepticism and questions from experts. Covaxin is an important milestone in vaccine discovery, for science and national fight against coronavirus". Covid-19 vaccines in India have been approved for restricted use in an emergency, based on limited data indicating that the potential benefits of the vaccine are greater than the risks. The government's operational guidelines for Covid-19 vaccines describe the protocol for reporting and investigation of severe (e.g., anaphylactic shock) and serious AEFI (e.g., long-term disability, hospitalisation, or death). The guidelines emp India's cumulative COVID-19 vaccination coverage near 8 crore mark

India's cumulative COVID-19 vaccination coverage administered 79 million $(7,91,05,163)$ vaccine doses across the country as of 4 April 2021 (Figure 4).

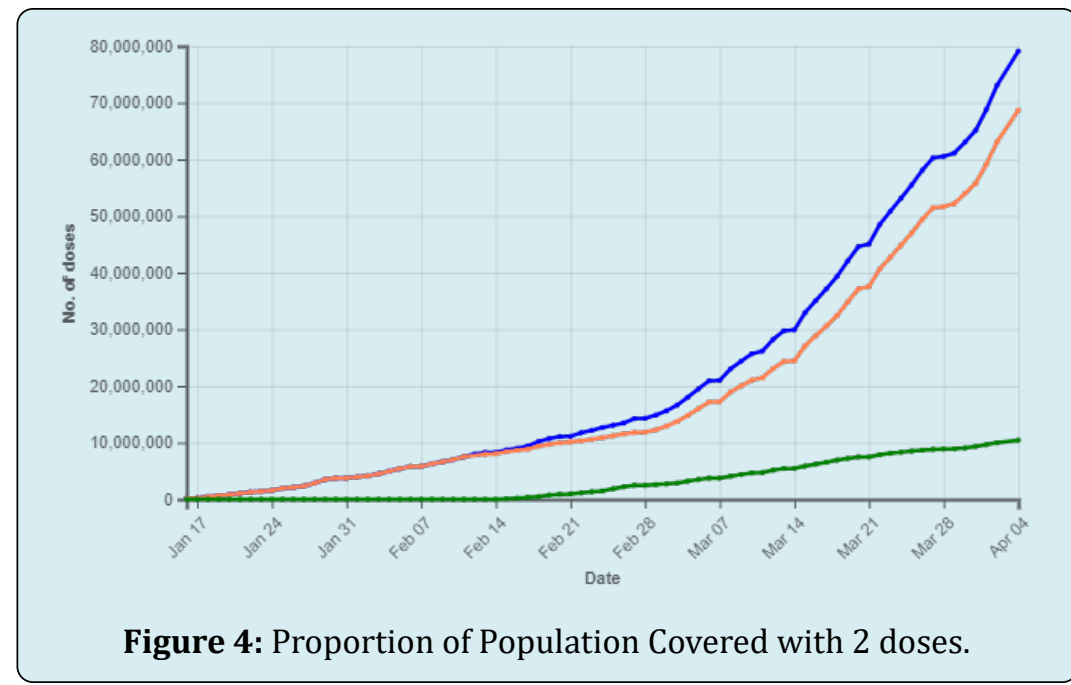

Indian fight against Covid-19 by crossing six crore vaccination on 1 April 2021. (State-wise coverage annexed) According to the health ministry statement, at least $6,02,69,782$ vaccine doses have been administered through $9,85,018$ sessions, as per the provisional report. These include 81,52,808 Health Care Workers (1st dose), 51,75,597 HCWs $\left(2^{\text {nd }}\right.$ dose $), 88,90,046$ Front Line Workers $\left(1^{\text {st }}\right.$ dose $)$ and 36,52,749 FLWs ( $2^{\text {nd }}$ Dose) $, 66,73,662$ beneficiaries aged more than 45 years with specific co-morbidities ( $1^{\text {st }}$ Dose) and 2,77,24,920 beneficiaries aged more than 60 years. Eight 
states - Kerala, Madhya Pradesh, Karnataka, West Bengal, Gujarat, Uttar Pradesh, Rajasthan, and Maharashtra account for 60 per cent of the cumulative vaccine doses given so far. Each of the eight states has administered more than 30 lakh doses. As seen in the annexure the coverage with 2 doses by states is in the range of $1-2 \%$. The best coverage of $3.6 \%$ and $2.7 \%$ in Lakshadweep and Ladakh small Union Territories. In the larger states Delhi has $1.98 \%$ coverage and Rajasthan $1.5 \%$. Despite boasting of 100 million vaccinations as of 10 April 2021 India has a long haul to cover. In the recent weeks we also are hearing about vaccine shortage and demand for covering younger age cohorts. Experts opine that opening vaccines for wider population will be a gamble with lives as the main aim of the vaccination is to reduce deaths and hospitalization. If opened the current vaccine production capacity will not meet the demand and even if it is augmented by financial support, it may take another 6-12 months to meet the demand.

Other countries seeking to protect their populations from the pandemic should draw at least three lessons from India's efforts.

\section{Challenge of Registration \& Service Provision}

Currently in India's digital platform called Co-win is being used to register beneficiaries that can provide forenoon and afternoon slot options. Registrations started for people for priority group of Health and other front-line workers, 60 plus age and those over 50 years with comorbidities. However initial days saw lots of problem in registration, scheduling and recovering the names registering and sometimes even mismatch with the documents produced.

From 1 April 2021 "Anyone aged 45 or above can start registering on Co-Win website as changes are reported to have already been made to the system. Those scheduling appointments in this age group for April 1, or after that, will not be required to carry a comorbidity certificate. Though, the objective is to encourage more and more online appointments, what is happening is more direct walk-ins at vaccination centres including my own example. I got an appointment for $30^{\text {th }}$ March 2021 online but could walk in get my slot for 11 March itself. I reached at 2pm (part of the wider afternoon slot) and had to wait till $1600 \mathrm{hrs}$ in a crowded queue for hours to get the shot. A narrower 1pm$2 \mathrm{pm}$ slot, instead, can improve the experience and fear of catching infection while waiting for our turn. The wider "forenoon" and "afternoon" slots have caused issues as people were not being offered a more specific time window to visit a vaccination centre. Vaccination slots on Co-Win will be fine-tuned to have hourly and two-hourly slots from the current wider "forenoon" and "afternoon" windows to benefit beneficiaries booking prior appointments, assures a health ministry official who heads the committee overseeing the vaccine delivery system, on Saturday 27 March 2021. Despite such assurances the ground level realities have not changed much till this report. "Once we reach a higher scale of vaccination, the system will automatically start finetuning slots to hourly and two-hourly windows. Fine-tuned time slots are also likely to reduce crowding at vaccination centres and chances of people being turned away despite prior registration.

Of the over 70 million registrations till $9 \mathrm{pm}$ on 3 April 2021, just 10 million were done online, whereas 37.5 million people walked in to receive the jabs, official data [3]. The total also includes about 2.3 crore health and frontline workers whose details were pre-populated on the Co-Win software. "Walk-ins can create uncertainties at times, as this process depends on availability of sufficient walk-in slots on a particular day. However, pre-registering and booking appointments online for specific time slots takes care of such issues and ensures a smooth, hassle-free process. With the addition of the 45-plus age group, the country is likely to reach 50 lakh vaccinations per day after April 1. The average number of people receiving the jab daily currently stands at 25 lakhs. The beneficiaries from April will also include those who are up for their second dose (especially Covaxin). The increase will closely depend to states' capacity and planning. But Co-Win is indeed prepared to handle so many vaccinations per day," a health ministry official claim. Despite the frustration in registering and scheduling the slot with minimum risk of being in crowd, the digitally enabled vaccine delivery in India deserves appreciation.

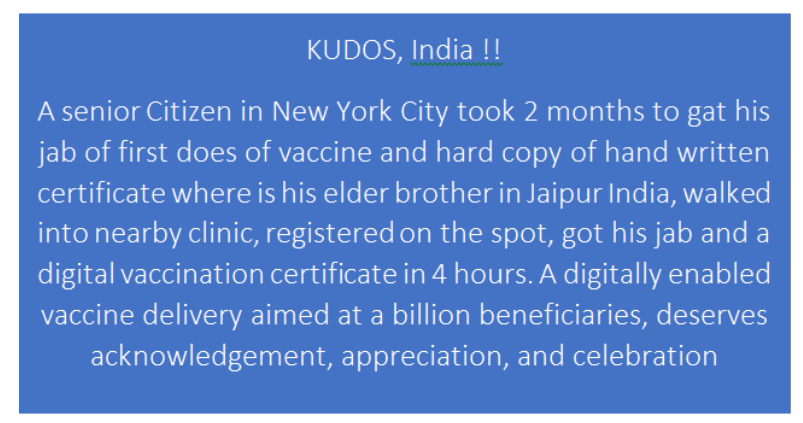

The other challenge faced with the software is its glitches of inability to provide vaccination certificates and the demand for the same increasing for all international and some domestic travels.

\section{Vaccine Wastage}

Vaccine wastage is linked to vaccine usage, which is the proportion of vaccines administered against vaccines issued to a vaccination site. The vaccine wastage rate is defined 
as 100 minus the vaccine usage rate. And the wastage rate directly determines the "wastage factor" that needs to be established for each vaccine in the immunisation schedule to accurately plan vaccine needs. The type of vaccine vials, the dosage and the quantity that can be extracted each time, based on the type of syringes used determines the vaccine wastage. Vaccine wastage is one of the key factors to be considered for vaccine forecasting and need estimation. Vaccine wastage is broadly divided into two categories: wastage in unopened vials, and in opened vials. Wastage in unopened vials can occur due to six broad reasons: if the expiry date has been reached; if the vaccine is exposed to heat; if the vaccine has been frozen; breakage; missing inventory and theft; and while discarding unused vials returned from the vaccination site. Wastage in opened vials can occur due to five broad reasons: while discarding remaining doses at the end of the session; not being able to draw the number of doses in a vial; submergence of opened vials in the water; suspected contamination; and poor vaccine administration practices. At the vaccination site, the wastage of vaccines has a direct relationship with session size, the number of beneficiaries per session and vial size.

The U.S. government provided piled up syringes to administer COVID-19 vaccines. The Pfizer vial originally intended for five 0.3 -milliliter $(\mathrm{ml})$ doses or $1.5 \mathrm{ml}$ total, contained $2.25 \mathrm{ml}$ of vaccine once the liquid is diluted for injection. Moderna vials intended for $10 \times 0.5-\mathrm{ml}$ doses, $5 \mathrm{ml}$ total, have $6 \mathrm{ml}$. The standard vaccine syringes supplied hold $3 \mathrm{ml}$., or 1-ml. syringe is often used for vaccines with small doses, like Pfizer's and Moderna's initially, that were not effective at extracting all available shots from the multidose vials supplied by vaccine makers resulting in vaccine wastage. This excess vaccine-up to two extra doses in Pfizer's fivedose vial and Moderna's 10-dose vials can be saved. Getting those extra doses out requires some skill-and the right syringe. Therefore, USA replaced them with narrower, smaller syringes specially designed "low dead-volume" syringes, which come in both sizes, that have plungers that slide all the way down to the needle to eliminate most of this trapped liquid. McKesson, the company that distributes the vaccine kits for the federal government, created "megasupply kits" for the Pfizer vaccine with syringes and needles that would allow the use of all six doses in January 2021, that reduced the demand for low dead-volume syringes [4].

The wastage accounts for 0.2 per cent in Canada based on Ontario's total vaccine inventory, as of 2 March 2021 [5]. Health authorities suspect that they would see up to $10 \%$ of vaccine wastage from the UK's programme [6].

India on the other hand has a vast experience of using auto-disable syringes in the national immunization program since early 1990's and the dose of all the vaccines was $0.5 \mathrm{ml}$ and so is the locally produced Covid 19 vaccines that suits the available $\mathrm{AD}$ syringes most of them made in-country. Covid 19 vaccination guidelines expect 100 persons to be covered per day, meaning they can use 10-11 vails per session. From a vial of ten doses, supplied by Government of India one can take out 11 doses. These 2 factors have minimised the vaccine wastage in India [7]. In India Over all wastage has been about $2.1 \%$ (Figure 5).

\begin{tabular}{|c|c|c|}
\hline \multicolumn{3}{|c|}{ VACCINE WASTAGE: THE STATES } \\
\hline Telangana & 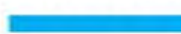 & $17.6 \%$ \\
\hline Andhra Pradesh &  & $71.6 \%$ \\
\hline UttarPradesh & $\bar{\square}$ & $9.4 \%$ \\
\hline Karnataka & $=$ & $6.9 \%$ \\
\hline Jammu \& Kashmir & - & $6.6 \%$ \\
\hline National average & 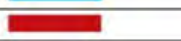 & $6.5 \%$ \\
\hline Rajasthan & 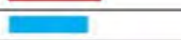 & $5.6 \%$ \\
\hline Assam & 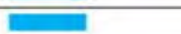 & $5.5 \%$ \\
\hline Gujarat & $=$ & $5.3 \%$ \\
\hline West Bengal & 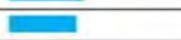 & $4.8 \%$ \\
\hline Bihar & $=$ & $4 \%$ \\
\hline Tamil Nadu & $=$ & $3.7 \%$ \\
\hline \multicolumn{3}{|c|}{ Figure 5: Vaccines Wastage. } \\
\hline
\end{tabular}

Human errors at a manufacturing plant forced Johnson \& Johnson to throw out 15 million doses of its COVID-19 vaccine that could have vaccinated $7 \%$ of the U.S. adult population [8]. Vaccine manufacturing is complex, with many potential points for errors. The multiple layers of quality checks by the producer and external inspectors throughout the process are essential to protect public health.

In view of the limited production of Covid 19 currently worldwide, all countries must examine why the vaccine is being wasted and build up a mechanism where this is being monitored, because by wasting the doses of the vaccine, we are denying another beneficiary's right to get vaccinated.

\section{After Effects Following Immunization (AEFI)}

Any untoward medical occurrence that follows immunisation and which does not necessarily have a causal relationship with the usage of the vaccine is called after effect following immunization (AEFI). The adverse event may be any unfavourable or unintended sign, abnormal laboratory finding, symptom or disease". Adverse reactions to a vaccine are broadly categorised based on their cause, severity, and frequency. The categories based on severity and frequency are: (a) Common minor reactions and (b) Serious and severe vaccine reactions. The cause-specific reactions can be vaccine product-related reaction and vaccine quality defectrelated reaction. 
The Vaccine Adverse Event Reporting System (VAERS) of USA has reported about 2-5 cases per million population vaccinated have reported Anaphylactic reactions mostly within 30 minutes of vaccinations. Over 167 million doses of COVID-19 vaccines were administered in the United States from the day of start December 14, 2020, through April 5, 2021 out of 167 million doses administered VAERS has received 2,794 reports of death $(0.00167 \%)$ among people who received a COVID-19 vaccine. A review of available clinical information including death certificates, autopsy, and medical records revealed no evidence that vaccination contributed to patient death. (https://www.opb.org/ article/2021/04/07/how-is-the-covid-19-vaccinationcampaign-going USA)

European Medicines Agency (EMA) reports that European countries like Germany, France, Italy, and Spain suspended use of AstraZeneca's COVID-19 vaccine following reports of dangerous blood clots in some recipients, though the evidence for the same is yet to be proved. The EMA investigating some 44 cases of rare brain clots and 14 deaths from more than 9 million doses of the vaccine possibly reports an association to the vaccine as of March 22, including 14 deaths. (https://qap.ecdc.europa.eu/ COVID-19/vaccinetracker.html) UK with a total of $31,622,367$ people $(60 \%$ of adults) a first dose, and 5,496,716 (10.4\%) with both shots. Of the 18.1 million people who have had the Oxford vaccine in the UK, 30 people have developed blood clots and seven had died as of 24 March though causal association is yet to be proved by the authorities. (https://news.sky.com/story/).

AstraZeneca's formula is one of three vaccines in use on the continent. But the escalating concern is another setback for the European Union's vaccination drive, which has been plagued by shortages and other hurdles and is lagging well behind the campaigns in Britain and the U.S.

In India AEFI monitoring is being carried through existing vaccine safety surveillance mechanism under the Universal Immunisation Programme (UIP). The National Adverse Events (Table 3).

\begin{tabular}{|c|c|c|c|c|c|}
\hline \multicolumn{7}{|c|}{ Times since last vaccine dose } \\
\hline AEFI* & $<\mathbf{3}$ days & $\mathbf{4 - 7}$ days & $\mathbf{8 - 2 8}$ days & $>\mathbf{2 8}$ days & Total \\
\hline Deaths & 93 & 18 & 11 & 0 & 124 \\
\hline Hospitalisation & 276 & 15 & 13 & 1 & 305 \\
\hline Severe AEFI & 55 & 4 & 4 & 0 & 63 \\
\hline
\end{tabular}

Table 3: Distribution of AEFI case by Time after last dose.

Following Immunisation (AEFI) Committee meeting on 31 March 2021 India reviewing 700 severe adverse events and 180 deaths are reported across the after COVID-19 vaccination, but none of them has been linked to the vaccine by the committee. The dissatisfaction among public health professionals is about the transparency as the findings of only two of these deaths have been made public so far.

\section{Adverse events in India, EU, UK Bear Striking Similarities}

Many of the AEFI and deaths reported in India bear striking similarities to those recorded in the European Union and the UK, of exceedingly rare cases of blood clots combined with low levels of blood platelets occurring within 2 weeks of vaccination". India has administered over 90 million doses, of which the bulk have been Covishield, the jab AstraZeneca licensed to Serum Institute. The analysis indicated that $90 \%$ of AEFI hospitalisations (276/305), more than $87 \%$ of cases of severe AEFI (55/63) and 93 out of 124 deaths happened within three days of the vaccination. While it is possible that events after a day to two could be missed out according to some public health activists. Out of 86 such events and 18 deaths reported in the EU and UK out of 25 million people's vaccination.

\section{- Bengaluru reports a case of VIPIT linked use of Covishield vaccine:}

An 80-year-old man, who had taken the first dose of Covishield on March 9, had later suffered a brain bleed, and subsequently slipped into a coma, died on 6 April 2021 at a private hospital. He had no comorbidities. State AEFI committee members including the chairperson had visited the 80-year-old man in hospital and had interacted with his family and treating doctors. This is the ninth death post vaccination in the state. As with six previous deaths, the state government insisted that it was not related to the vaccine and it was just coincidental. The recent deaths of two elderly women, one each in Ramanagar and Chikkaballapura districts of Karnataka, are yet to reflect in the state's AEFI data.

A renowned neurologist suspects it to be a case of vaccine-induced prothrombotic immune thrombocytopenia (VIPIT), which the UK also report comes with using Covishield. VIPIT is a condition where platelets decline post 
vaccination. No postmortem was done as per the family's decision, a challenge to understand the mechanism.

Given the size of the country and number of vaccination centres across the country, tracking AEFI reported is a challenge and the committee would have to work much faster to do proper causality assessment.

\section{Vaccine Hesitance}

India's Universal Immunisation Programme and Pulse Polio campaigns have encountered vaccine hesitancy for years stemming from various causes. A December 2018 study across 121 high priority districts chosen by the Health Ministry revealed that nearly a quarter of parents refused to vaccinate their children under the belief that vaccines caused more harm than good. Another study inferred that several notable political leaders have also worked to erode trust in the scientific community.

Despite having thousands of data points proving the vaccine's safety, a few unanswered questions are creating a data deficit that the purveyors of misinformation are using to their advantage.

There is a much higher level of vaccine hesitancy in ethnic minorities, those living in deprived areas and those that distrust the NHS. There is an urgent need to tackle the overwhelming misinformation about COVID-19 that is leading to this uncertainty and confusion about the vaccines is a high risk of unequitable roll out of the vaccination programme in the UK.

A recent study conducted by Praveen et al., showed that only $35 \%$ of the Indian population has positive sentiments regarding the vaccine, which is a concern for the government and policymakers. The study revealed that many Indian citizens still feel that the whole pandemic is exaggerated, which has also affected the vaccination programme. Moreover, wariness about vaccine trials and health afterwards, fear of death from the vaccine, allergic reactions to the vaccine, distrust over pharmaceutical companies, doubts about data provided by vaccine companies, the prevalence of several vaccines and concerns over choosing the safest, and the rush in providing vaccine were among the other concerns expressed by Indian citizens.

A survey conducted in January 2021 by Local Circles, of 17000 people, found that vaccine hesitancy was at 62 percent. Of the hesitant lot, 59 percent were worried about the side-effects of the vaccine. An in-house study conducted by Bangalore Baptist Hospital found that female staffers faced more side effects following vaccination than their male counterparts. The cohort comprised 218 employees who had taken their first dose of Covishield. Most of the employees are women (172). Side effects were seen in 75.5 per cent of the women and 45 per cent of the men. The most common ones were pain at the injection spot (76.1 per cent), body ache (46.8 per cent), headache ( 30.3 per cent) and fever (22 per cent). The respondents also complained of stiffness in the arm (11.5 per cent), swelling in the arm (16.5 per cent) and GI and vomiting (4.1 per cent). But there was no case of serious adverse event following immunisation (AEFI).

Miscommunication on social media the post said, "26 years old from India died suddenly after getting the COVID-19 injection,", omitting the fact that the time difference between the vaccination and her death was over a month. Viral messages on Facebook, Instagram and WhatsApp said that "she died after taking the vaccine". The fact was that she had received the first dose of the vaccine on 5 February, nearly a month before she fell ill, and doctors, in their initial investigation, have ruled out the COVID-19 vaccine as the reason behind the death.

Globally, the use of mRNA technology in the making of Pfizer and Moderna COVID-19 vaccines have also raised concerns and created a space for misinformation and conspiracy theories. Many anti-vaxxers believe that the mRNA vaccines will alter people's DNA and are made to depopulate the earth. Scientists, who have studied the technology, have maintained that the COVID-19 vaccine cannot alter human DNA. Therefore, there is a need for clear communication and transparency from vaccine manufacturers, which will help in quashing misinformation at the source $[9,10]$.

Vaccine hesitancy is a continuum of behaviours ranging from delay in receipt to vaccination refusal. A recent study in five LMICs- Mothers of children in Sirajganj, Bangladesh ( $n=60)$, Shanghai, China ( $\mathrm{n}=788)$, Addis Ababa, Ethiopia ( $\mathrm{n}=341$ ), Guatemala City and Quetzaltenango, Guatemala $(\mathrm{n}=767)$, and Chandigarh, India $(\mathrm{n}=309)$, completed a survey between 2016 and 2018 using the WHO's 10-item Vaccine Hesitancy Scale. Compared to mothers in China, mothers in Bangladesh perceived less vaccination benefit $(\beta: 0.56, P=0.0001)$, however, mothers in Ethiopia ( $\beta:-0.54$, $P<0.0001)$ and Guatemala $(\beta:-0.74, P=0.0004)$ perceived greater benefit. Education level was not significantly linked with vaccine hesitancy. Local circumstances are important to consider when developing programs to promote vaccines [11].

There is little that can be done to allay the doubts of the hardcore conspiracy theorists, in fact, all attempts to do so simply confirm to them that everyone is in on the conspiracy. But the others are worth understanding and acting upon. Part 
of the doubt about the vaccine comes from the unprecedented way it has been developed. 2020 was a great advertisement for science as it underlined its continued criticality to the world. But it also gave us a ringside view of exactly how untidy the scientific process is. Guidelines were changed frequently as scientists began to understand the virus and its effects better. Masks were initially thought to be useless and then recognised as the single most important instrument in the battle against Covid-19, miracle drugs were found and subsequently denounced, the directive to be on guard against contaminated surfaces led to a frenzy to hand and vegetable washing, which was later seen to be an overreaction. Even now, a lot of questions remain unanswered.

Usually all this happens inside laboratories and scientific journals and we, the lay public, do not see any of it. What matters is that what scientist have at the end of the process is a rigorously tested, safe and effective vaccine. The way some vaccines have communicated their progress has also done the effort no favours. AstraZeneca has scored self-goals and jeopardised the reputation of what otherwise looks like an excellent vaccine. The latest imbroglio around its claimed efficacy of $79 \%$, the public rebuke that it received from US regulators that it was sharing dated information and against the subsequent clarification that it was $76 \%$ only 48 hours later is impossible to comprehend.

The Indian experience of the same vaccine has fortunately been smoother. The local partner, charged with the responsibility of manufacturing for large parts of the developed world, has had its share of challenges, but has managed to deliver to the needs of the local market. The rollout too has been smooth enough, and compared to how its principals have acted, managed. The other problem comes from a tendency to privilege personal anecdotal experience over large-scale impersonal data. Take the doubt sowed by instances of fully vaccinated people getting infected again. If vaccine efficacy is as high as $95 \%$, even then some people will get sick. Given the large base of those vaccinated, this small number in terms of percentage will translate into a large physical number. However, the emergence of such cases, particularly if they occur in people one knows of, is psychologically difficult to reconcile with the expectation of being protected. It does not help that these instances, along with many stories of sundry side-effects (gain hardly ever serious or unanticipated), get disproportionate play. Unlike the West, particularly America, where the anti-vaccination segment is a significant one, in India there is no organised attempt to challenge the idea of the vaccine. The problem in India is different - for one, inertia is a big barrier; witness the number of people who are casual about wearing masks correctly or distancing themselves socially. Then there are those that are full of doubts about the efficacy of the vaccine and the possibility of side-effects. There is a danger in believing that simply getting the vaccine to people is enough. The experience of the immunisation programme in India is clear about the need to communicate and to persuade those who are hanging back to get themselves vaccinated. Mere information dissemination is not enough; although clear and authoritative communication about the correct scientific picture is important, what is truly needed is a nuanced understanding of the barriers that holds people back and an appropriate framing that converts an information-heavy message into one that is motivating and persuasive.

We have a government at the Centre that is a communication sophisticate. It has an extremely effective communication ecosystem that it uses for its political ends. It is time that this network gets deployed to address a larger national need. In addition, the government needs to mount a large, thoughtfully constructed campaign to ensure that the different psychological barriers to vaccination are addressed. The soaring numbers that we are seeing today will continue to rise unless there is a serious attempt to get everyone vaccinated.

The researchers said that the exact reason for the higher incidence of side effects among women was not known. "It could be that women are more likely to report symptoms than men. Anxiety might be more in women; many side effects are related to anxiety. The female immune system is more reactive compared to men's, which is why autoimmune diseases are reported more among women. Hormonal differences may also be at play. The vaccine reactogenicity is more in the younger population than in the older, and this was seen in the cohort. Seventy-six cohort members are more than 40 years old and 142 are aged below 40 . Side effects were seen among 34 members aged below 40 and only in five aged above 40 . "The reason could be the reaction of the immune system, which is more active in the young. The phase 3 trials of Covishield also said that younger people had higher reactogenicity," she added.

In Karnataka, 27 people have experienced serious AEFI so far. Twenty-one of them are women who work as healthcare and frontline workers. "Most of the healthcare workers are women. That could be one reason for more instances of AEFI among women. But we have not done any gender-based assessment of AEFI. No serious adverse events have been seen among senior citizens so far reports the Director National Health Mission of Karnataka.

Despite the government running an intensive awareness campaign to remove vaccine hesitancy and standing firm endorsing safety and efficacy of both the vaccines, states like Chhattisgarh refused supplies of Covaxin, partly, it is felt by the Centre. 


\section{Debate on Interval between 2 doses:}

But there are two big hurdles for world-wide vaccination drive. First, the vaccine supply is not enough. The second constraint is self-imposed, by giving the two vaccine doses too close to each other. The two doses of Covishield are given 6-8 weeks apart, and Covaxin the interval is 4-6 weeks.

The UK was in the same quandary in December. Its new variant had caused an explosion of infections. That is when it decided to take the "first-dose-first" approach, trusting that "some protection for all" would be better than "full protection for some". What appeared controversial, was not a desperate gamble as the results showed that the UK made the right call. Since then, some other countries have also delayed their second doses to focus on the first. Germany has adopted the 12-week interval for the AstraZeneca vaccine (Covishield).

Recently Canada and Spain have opted for all second doses after 4 months.

A vaccine needs time to build immunity. For the first two weeks after vaccination, its effect is negligible. But then, it starts increasing. However, you should not delay the second dose too long because then the first dose's effect starts waning. The challenge is to find the sweet spot where the effect of the first dose peaks, so the second dose can raise it further. AstraZeneca itself had recommended a 4-12week gap. "A 12-week gap would not be long enough for that initial protection to fade," When AstraZeneca published trial results last November, some volunteers who were "mistakenly" given a half dose first were found to have better immunity than those who got two full doses. The consensus today is 12-week gap is better.

India makes 200 million doses of vaccines over two months; we cannot vaccinate 20 crore people with them but only about 10 Crore people. Then question arises as to how much better?

In March 2021, The Lancet published data that showed the UK had made the right choice. It said a single dose of the AstraZeneca shot had $76 \%$ efficacy from the start of the 4 th week to the end of the third month, "and antibody levels were maintained during this period with minimal waning." Another report in Stat says a single dose of mRNA vaccines from Pfizer-BioNTech and Moderna confers 80\% protection "two weeks after an initial shot," rising to $90 \%$ after the second dose. The AstraZeneca vaccine's efficacy rose to $81.3 \%$ with a 12 -week gap, as compared with $55.1 \%$ after a 4-week gap. Among participants who were younger than 55 years, the antibody response was "more than two-fold higher (for) a dose interval of at least 12 weeks than an interval of less than 6 weeks[13]
Some doubts remain as some critics of the longer gap say it "may be less effective for older people, whose immune response to the vaccines is typically weaker than in younger people," according to the Stat report. Professor N K Ganguli, Retired Director of ICMR India argues that the speed at which the population in India is being vaccinated is still lagging and precious time is being wasted by not broadening the vaccine eligibility criteria in the race against the wily virus. He asserts that a partially vaccinated population also puts pressure on the virus to mutate and find more susceptible hosts compounding the problem. There are worrisome reports coming in of people getting infected after 14 days of the second dose of vaccination albeit with a less serious disease pattern (perhaps with a variant). They could then spread further. India has already vaccinated many senior citizens. As for people forgetting their second vaccine date, a fix can be found for that. But the more pressing concern now is to increase vaccination rapidly. For that, a longer gap might be necessary.

If we must vaccinate at least $60 \%$ of our 1,350 million population in the next 6 months for herd immunity to develop, we need to vaccinate 4 million people a day which translates into 50,000 centres delivering 80 doses each per day. The private sector should be roped in for vaccination delivery as well and vaccine wastage should be decreased. In case there is not much of a turnout a parallel line should be created to vaccinate the younger people.

This is the time to ramp up our genetic sequencing programme to understand more about the variants. We also need to assess the efficacy of existing approved vaccines against the new variants. Other vaccines like Novavax (protein subunit vaccine) and Johnson \& Johnson vector vaccine which have demonstrated higher efficacy against variants with E484K mutation must be approved on an emergency use basis, possibly without the local trials. These measures will help in Vaccine production ramping up.

It is also a time for the states and the Centre to come together and formulate health policies in the best interest of India's population, leading to another debate on if Covid 19 vaccination should be free across the country

\section{Debate on If Vaccination be given Free to all:}

The Supreme Court on 30 April 2021 asked GOI why it could not vaccinate the entire Indian population against Covid-19 free of cost under the National Immunisation Programme, On the two Indian vaccine manufacturers, the pricing of Covid-19 vaccines manufactured by all manufacturers is an extremely important issue and financial assistance or grant given to the companies by the Centre for research and developing the vaccines is equally important. 
The private firms should not decide what quantity of vaccines it would send to a particular state, as the private firms cannot acquire equity rights over public good. Universal free of cost immunisation in comparison with the present policy of $50 \%$ supply to GOI to vaccinate frontline workers and those above 45 years and the rest at a higher price to states and private hospitals may exclude marginalised sections of society [13].

\section{Conclusion}

Immunization is a global public good. No vaccination is $100 \%$ fool proof. The aim of Covid 19 vaccination is reducing the proportion deaths and hospitalization.

All vaccines are equally good, their current reported efficiency and effectiveness depends upon when they were tested, and what size of the population. People should accept whatever vaccine is locally available.

The current vaccine production worldwide is not enough to open for all age population. Therefore, prioritizing the population-based on age, gender, and other profiles of cases in is important.

The trails of vaccines in pregnant women and children need to be pursued. Existing vaccination coverage in no country is adequate to arrest the second wave of infection, but will prepare ground for preventing the third wave later in 2021. The global aim should be to bring countries together, to dispel the temptations of isolationism and nationalism, and to address the challenges that could only be achieved together in the spirit of solidarity and cooperation, namely peace, prosperity, health, and security.

We are not sure if the current vaccines can reduce infection, but surely, they will minimize the damages the infection causes.

Despite the government running an intensive awareness campaign to remove vaccine hesitancy will continue to bogdown people to be reluctant or refuse in getting vaccinated.

\section{Way Forwards}

- Every country must be committed to ensuring universal and equitable access to safe, efficacious, and affordable vaccines, medicines and diagnostics for this and future pandemics.

- We must move forwards to optimally use our vaccine production capacity and accelerate of our vaccination efforts and immunize all eligible population specially marginalised sections of society

- We must pursue micro-containment of area by area with cases.

- It is vital that even after vaccination with both doses everyone must wear masks, practice social distancing, and wash their hands when around those who have not been vaccinated

\section{References}

1. Krishna Uday Kumar (2020) director of Duke Global Health Innovation Center.

2. (2020) Rupali.Mukherjee@timesgroup.com, Bengaluru Edition.

3. (2021) Walk-ins for shots four times the no. of online registrations.

4. (2021) Syringe size and supply issues continue to waste COVID-19 vaccine doses in United States, Sofia Moutinho.

5. At least 1,500 COVID-19 vaccine doses wasted in Ontario.

6. What happens with unused Covid vaccines?

7. (2021) Understanding Covid-19 vaccine wastage in India.

8. (2021) Why Johnson \& Johnson throwing out 15 million COVID-19 vaccine doses.

9. Dickerson J (2020) COVID-19 vaccine hesitancy in an ethnically diverse community.

10. (2020) Data Deficit Around Deaths Post COVID-19 Vaccine Creates Hesitancy, Abhilash.

11. Wagner AL, Masters NB, Domek GJ, Mathew JL, Sun X, et al. (2019) Comparisons of Vaccine Hesitancy across Five Low- and Middle-Income Countries. Vaccines 7(4): 155.

12. Should India delay 2 nd vaccine dose to avoid 3rd Covid wave? Abhilash.Gaur@timesgroup.com TOI, Bengaluru $1 / 5 / 21$

13. Dealing with The Second Wave, NK Ganguli \& Namita Jaggi, TOI Bengaluru edition 1 May 2021

14. Why can't all get free jabs under nat'l plan? Dhananjay. Mahapatra@timesgroup.com New Delhi 